\title{
The geometric module in the rat: Independence of shape and feature learning in a food finding task
}

\author{
PATRICIA L. WALL, LEIGH C. P. BOTLY, CHRISTINA K. BLACK, and SARA J. SHETTLEWORTH \\ University of Toronto, Toronto, Ontario, Canada
}

\begin{abstract}
Rats found food in a rectangular enclosure in three experiments testing how learning about a distinctive feature near a goal interacts with learning based on the geometry of an enclosure. Rats trained to follow a feature in square and triangular enclosures and to use geometry in the rectangle followed the feature when it was in the rectangle (Experiment 1). Rats trained with the feature in a geometrically consistent corner of the rectangle learned about both geometry and the feature (Experiment 2). Training with the feature in the square did not block learning of geometry when both predicted the location of food in the rectangle (Experiment 3). The "geometric module" (Cheng, 1986) may have a special status in spatial learning.
\end{abstract}

A specific geometric module for encoding the macroscopic shape of the environment was proposed by Ken Cheng (1986). Cheng trained rats in a working memory paradigm to search for food in a rectangular box. On each trial, the food was first exposed in a novel location, and $75 \mathrm{sec}$ later the rat was allowed to search for food buried in a corresponding location in an identical box. When there were no other cues, rats searched primarily in the correct location and the diagonally opposite (i.e., geometrically equivalent) location. Remarkably, rats confused geometrically equivalent locations even in the presence of cues such as panels in the corners of the rectangle that could disambiguate geometric information. It was primarily this apparent impenetrability of geometric processing to other kinds of information that led Cheng to suggest that the processing of local spatial geometry is modular, sensu Fodor (1983). Margules and Gallistel (1988) went on to show that Cheng's finding depends on rats' being unable to locate themselves in the box by means of dead reckoning or cues outside it. Because the rat must be disoriented and/or the box must be reoriented between trials, Cheng's findings indicate that local geometry is primary in establishing reorientation in familiar environments.

Domestic chicks (Vallortigara, Zanforlin, \& Pasti, 1990), pigeons (Kelly \& Spetch, 2001; Kelly, Spetch, \&

This research was supported by a Research Grant to S.J.S. from the Natural Sciences and Engineering Research Council of Canada (NSERC). Parts of Experiments 1 and 2 were conducted with the support of an Undergraduate Summer Research Award from NSERC to the first author. The second and third authors contributed equally to Experiment 3. We thank Amanda McCleery for assistance with testing animals, Andy Gristock for animal care, Brett Gibson for help with statistics, and Ken Cheng and Jennifer Sutton for comments on the manuscript. P. L. Wall is now at the University of Western Ontario. Correspondence may be directed to S. J. Shettleworth, Department of Psychology, University of Toronto, 100 St. George St., Toronto, ON, M5S 3G3 Canada (e-mail: shettle@psych.utoronto.ca).
Heth, 1998), Xenotoca eisenii, a species of fish (Sovrano, Bisazza, \& Vallortigara, 2003), rhesus monkeys (Gouteux, Thinus-Blanc, \& Vauclair, 2001), and human children (e.g., Hermer \& Spelke, 1996) have been tested as Cheng's rats were tested (review in Cheng \& Newcombe, in press). All have been found able to use the shape of an enclosure alone to reorient, but it is not clear whether the relative control by geometry as compared with local features (single distinctive walls or distinctive cues in the corners) differs across species. Researchers (e.g., Gouteux et al., 2001) have not always acknowledged that exclusive reliance on geometry was confined to the working memory paradigm in Cheng's (1986) Experiment 1, in which the location of the food changed for every trial. When the food was in the same place trial after trial, in the reference memory paradigm of his Experiments 2 and 3 , disoriented rats did eventually learn to use local features. For example, when one long wall had a distinctive brightness in the reference memory task, rats reached a criterion of 9 out of 10 successive trials correct within 19-89 trials. When, instead, each corner of the box was marked by a visually and olfactorally distinctive panel, the same rats met criterion within 14-129 trials. Tests with some panels removed or moved relative to the geometry of the enclosure showed that the rats were primarily using the panel closest to the goal (Cheng, 1986, Experiment 3).

The cited articles on rhesus macaques, pigeons, fish, and chicks report only data from reference memory tasks. For example, the macaques (Gouteux et al., 2001) had 50 trials with the food in one place. All these studies show that, like rats, the animals tested eventually learned to use local features of an enclosure when they were available to disambiguate geometry. Studies with human children and adults (e.g., Hermer \& Spelke, 1996; review in Cheng \& Newcombe, in press) have more closely approximated the conditions of Cheng's original demonstration that geometry predominates over more informative local cues in a working memory task. Individual subjects in 
those studies have generally been tested on only a few trials. Therefore, it is of considerable interest that whereas human children disregard featural information as a cue for reorientation under some conditions, adults in the same studies use featural information to improve performance (e.g., Hermer \& Spelke, 1996).

The circumstances that allow humans to use local features instead of geometry in reorienting have received a great deal of attention (e.g., Hermer \& Spelke, 1996; Learmonth, Nadel, \& Newcombe, 2002; Learmonth, Newcombe, \& Huttenlocher, 2001; Wang, Hermer, \& Spelke, 1999). It has been suggested that, over the course of development, children learn to use language to combine geometric and nongeometric information (HermerVazquez, Moffet, \& Munkholm, 2001). However, even very young children can reorient by means of nongeometric information in some conditions (e.g., Learmonth et al., 2002; Learmonth et al., 2001). Learmonth et al. (2002) argue that the small enclosures in which much of the work with children has been conducted impair spatial navigation by minimizing movement. Increasing the size of the experimental space improves the performance of 3- and 4-year-old children, enabling them to use both geometric and local features (Learmonth et al., 2002).

Despite the explosion of research on how geometry and visual feature learning interact in other species, there has been little further work on this question with rats. The research of Pearce and his colleagues (Hayward, McGregor, Good, \& Pearce, 2003; Pearce, WardRobinson, Good, Fussell, \& Aydin, 2001) is one of the few exceptions (see also Benhamou \& Poucet, 1998; Golob, Stackman, Wong, \& Taube, 2001; Golob \& Taube, 2002). Pearce et al. (2001) pointed out that Cheng's (1986) claim for a specialized geometric module has important implications for the debate (see Mackintosh, 2002; Shettleworth, 1998) over whether spatial learning follows the same principles as associative learning does. In associative learning, exemplified by Pavlovian and instrumental conditioning, different sources of information such as lights and tones can be seen as competing for a limited pool of associative strength (Rescorla \& Wagner, 1972). Such competition is evidenced in the phenomena of overshadowing and blocking. In overshadowing (Pavlov, 1927), less is learned about a given cue (the target), when it is trained in compound with a second cue than when it is trained alone; the target is said to be overshadowed. In blocking (Kamin, 1969), first training a single cue to near asymptote diminishes (i.e., blocks) acquisition to a predictive target cue that is then compounded with it, as compared to a control with the target and the second cue present from the outset.

Overshadowing and blocking are found when landmarks and/or beacons are manipulated in some spatial learning paradigms (see the review in Chamizo, 2003). However, Cheng's (1986) discussion of the geometric module suggests that geometric information should not be blocked or overshadowed by landmarks or beacons (often referred to collectively in discussions of the geo- metric module as featural cues). And indeed, Pearce and colleagues (Hayward et al., 2003; Pearce et al., 2001) found no evidence that learning the geometry of an enclosure was blocked or overshadowed by such features. In those studies, however, rats performed a water escape task, usually in a triangular enclosure with a curved base. Thus, both the animals' motivation and, in most cases, the geometric cues available differed from those in other studies of the geometric module.

The experiments reported in this article were designed to examine further how rats integrate or fail to integrate geometric and featural information when searching for food in a rectangular enclosure like the one used by Cheng (1986). Experiment 1 was analogous to the studies with children and human adults in which subjects used verbal information acquired outside the test environment to disambiguate geometry (Hermer-Vazquez et al., 2001; Hermer-Vazquez, Spelke, \& Katsnelson, 1999). Rats were trained to use a black panel to locate food in two geometrically ambiguous enclosures, a square and an equilateral triangle, while concurrently learning to use geometry in a featureless white rectangle. The black panel was then presented in the rectangular enclosure in both geometrically correct and incorrect locations. In Experiment 2, we tested whether geometric cues would acquire control in the rectangle when the salient black panel was present from the outset; in effect it was a replication of Cheng's Experiment 2 under our conditions. Experiment 3 was a test of whether prior training with the black panel in the square enclosure would block learning of geometry in the rectangular enclosure. Thus it was a test of whether the independence of geometry and feature learning demonstrated in water escape tasks (Hayward et al., 2003; Pearce et al., 2001) extends to the situation in which the geometric module was originally demonstrated.

\section{EXPERIMENT 1}

\section{Method}

Subjects. The subjects were 8 experimentally naive male LongEvans rats 96-157 days of age and weighing 338-360 g at the start of the experiment. The rats were housed individually in plastic tub home cages ( $45 \mathrm{~cm}$ long $\times 25 \mathrm{~cm}$ wide) and were kept at approximately $90 \%$ of their ad-lib weights throughout the experiment by controlled feeding of Purina rat chow after experimental sessions. Water, nesting paper, and an opaque plastic tube "home" big enough for a rat to enter were always available in the home cages. The rats were maintained on a reversed 12:12 light:dark cycle and tested during the dark phase.

Apparatus. The three training environments were 50-cm-high collapsible fence-like enclosures made of white melamine: a rectangle $120 \mathrm{~cm}$ long $\times 60 \mathrm{~cm}$ wide (the size used by Cheng, 1986), a square $60 \mathrm{~cm}$ on a side, and an equilateral triangle $110 \mathrm{~cm}$ on a side. When in use, an enclosure was placed directly on the textured cement floor of the testing room, centered below a shaded $10-\mathrm{W}$ light that hung approximately $65 \mathrm{~cm}$ above the floor. This light provided the only illumination in the experimental room, which was about $2.5 \mathrm{~m}$ square. The placement of the shaded light ensured that the area surrounding the box was only dimly illuminated. During all training sessions, a metal bowl $4 \mathrm{~cm}$ deep and $8 \mathrm{~cm}$ in diameter at- 
tached to a heavy $10-\mathrm{cm}$-square $1-\mathrm{cm}$-thick white metal base was placed in each corner of the apparatus. The bowls were filled approximately $3 / 4$ of the way with the granular commercial bedding Bed-o'cobs (The Andersons, Maumee, $\mathrm{OH}$ ) mixed with two to three finely crumbled pieces of Froot Loops sweetened cereal. A single whole Froot Loop was used as a reward. During sessions with the square and triangular enclosures, a moveable black plastic corner panel was inserted into one of the corners of the apparatus (Figure 1). Each $50-\mathrm{cm}$-high panel fit snugly into the corner of an enclosure and spanned $10 \mathrm{~cm}$ on each wall on either side of the corner.

Pretraining. The rats received 1-2 days of pretraining in the home cages described under Subjects, during which they were shaped to dig for Froot Loops buried at the bottom of the metal bowls of bedding.

Training. Training consisted of alternating geometry and feature days in which the all-white rectangular enclosure was used for the geometry days and the square and triangular enclosures, each with its black corner panel, were alternated for the feature days (see Figure 1). Thus, training could be divided into blocks of 4 days consisting of 2 geometry days and 2 feature days. Each training day consisted of 8 trials separated by a 1-min intertrial interval (ITI). Before each trial, one whole Froot Loop was buried at the bottom of a randomly chosen bowl. The remaining bowls had no reward. In the rectangle, the rewarded bowl was placed in one of two predetermined geometrically correct corners. In the square or the triangle, the rewarded bowl was always in the same corner as was the black panel. The location of the rewarded corner was determined as described in the next paragraph.

Between trials, the rats were kept in opaque black travel boxes $(25.5 \mathrm{~cm}$ wide $\times 25.5 \mathrm{~cm}$ long $\times 20.5 \mathrm{~cm}$ high $)$ just outside the experimental room. Before each trial, the rats were disoriented by rotating the travel box on a lazy Susan approximately eight times in $1 \mathrm{~min}$. Disorientation occurred in one of the four corners of the experimental room, each used twice per day in random order. The orientation of the apparatus plus goal location also changed for each trial. There were eight orientations for both the rectangular and square enclosures and goal, four with the walls of the enclosure parallel to the walls of the room and four with them at $45^{\circ}$ to the walls of the room. Each orientation was used in a random order in each training session. There were 12 orientations for the triangular en- closure plus goal, eight of which were used in a random order in each training session. On a given day, all rats received the same sequence of apparatus positions and disorientation locations.

At the beginning of each trial, the rat was placed in a predetermined starting position within the apparatus. Both the rectangular and square enclosures had eight possible starting locations (in the center of the apparatus facing each of the walls, or in the center of each of the walls facing the center of the apparatus), each of which was used in a random order on each training day. The triangular enclosure had six possible starting positions, all of which were used during each training day in the triangular enclosure with two locations repeated for a total of eight trials.

During the first 4-day block of training, the rats were allowed to make as many choices as were necessary in order to find the reward. A choice was defined as a dig that displaced the surface of the bedding in a given bowl. If a rat failed to make a choice within 2 min during this first block of training, the reward was placed on top of the appropriate bowl and the rat was given $1 \mathrm{~min}$ to eat it. These correction trials were not included in the calculations of percent correct. During all later blocks, a rat was allowed to make a maximum of two choices per trial, after which it was removed from the apparatus. If a rat found the food on the first choice, it was allowed to eat and was then immediately removed from the apparatus.

In the rectangular enclosure, searches were scored as correct (C) if the rat chose the bowl containing the reward, as a rotational error (R) if it chose the corner $180^{\circ}$ from the correct bowl, as near $(\mathrm{N})$ if it chose the incorrect corner closer to the correct bowl, and as far (F) if it chose the incorrect corner farther from the correct bowl (see Figure 1). A choice was considered geometrically correct in either the absolutely correct location or its rotational equivalent ( $\mathrm{C}$ or $\mathrm{R}$ ). In the square and triangular enclosures, searches in the corner with the black panel were scored as correct. Training continued until the rats reached criterion, which was defined as 4 consecutive days of $75 \%$ accuracy or better on their first choice

Nonrewarded probes. Following criterion, the rats had one block of nonrewarded probe testing, in which one of the final four trials on each day was conducted with no Froot Loop buried in any of the bowls.

Testing. The animals were tested by combining the geometric and feature cues that had been presented separately during training.

Train

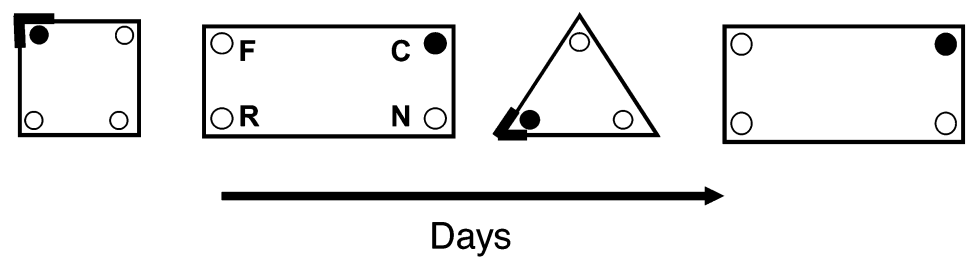

Test

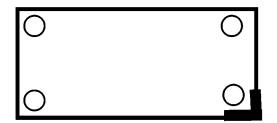

Conflict

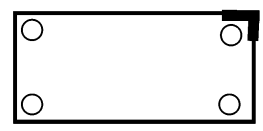

Consistent

Figure 1. Arrangement of the enclosures and corner panel for training and testing in Experiment 1. The top row depicts one block of training. The orientation of the enclosure + panel and rewarded bowl within the room was changed from trial to trial as described in the text. Filled circles $=$ bowl containing reward; open circles $=$ unrewarded bowls; $C$, correct choice; $R$, rotational error; $N$, near error; $F$, far error. 
Two different tests with the black panel in the rectangular enclosure were given in counterbalanced order. In the consistent test, the black panel was placed in one of the geometrically correct corners. In the conflict test, the panel was placed in a geometrically incorrect corner. No food was placed in any of the bowls, and the rats were given a single trial that lasted $1 \mathrm{~min}$, during which they were allowed to make as many choices as possible. Testing consisted of 2 test days separated by 2 retraining days. Each rat was assigned a disorientation location, as well as a starting position, which was used for both of the tests. Two retraining days ( 1 geometry and 1 feature day) were given between test days. The retraining days were arranged so that if the rat received a geometry day before the first test, it received a feature day before the second test, and vice versa.

Statistical analysis. Whenever there was a single test trial of a given kind, a binomial test was used to assess whether a particular corner was chosen by significantly more rats than would have been expected by chance. In experiments that included two or more tests of the same kind, the combined test results were analyzed using GLIM (Generalized Linear Interactive Modeling, Numerical Algorithms Group, Downers Grove, IL). GLIM fits a log-linear model to the data (Francis, Green, \& Payne, 1993). The log-linear model produced by GLIM specifies a binomial error term, as opposed to another type of distribution such as normal. The test statistic that GLIM uses to examine these effects was then compared with a chisquare distribution (note that this is not a chi-square test of independence). Alpha was set at .05 throughout for the determination of significant effects.

\section{Results and Discussion}

Two rats were dropped because they failed to meet criterion in the rectangle within eight blocks of training; a 3 rd rat was dropped for failing to meet criterion in any of the training environments within seven blocks. The remaining 5 rats all reached criterion within four to six (mean 5.4) blocks of training. None fell below criterion during the block of probe days. In previous studies related to this one, some rats have also failed to learn or have learned much more slowly than others. For example, in Cheng's (1986, Experiment 2) reference memory task, the slowest rat took more than three times as many trials to reach a learning criterion as did the next slowest one, and when the same rats were retrained for testing in Cheng's Experiment 3, 1 failed to meet criterion. Failures to learn have also been observed in working memory versions of the task ( 3 failures out of 10 rats in Margules \& Gallistel, 1988, Experiment 1; 3 out of 8 in Golob et al., 2001, Experiment 3.) As Golob and Taube (2002) pointed out, studies from which animals are dropped will tend to produce data that exaggerate the degree of control by geometry or features, as the case may be, over behavior in the whole population. No rats were dropped from our Experiments 2 and 3 for failing to learn the reference memory tasks.

To characterize final performance, data from the 4 days in which each rat reached criterion and the 7 regular trials in each of the 4 probe days were examined (i.e., 30 trials per rat in the rectangle and 15 trials per rat in the square and in the triangle). All rats made 11 or more correct choices in the triangle and in the square, more than twice the chance expectations of 5 or 3.75 correct choices, respectively. The results of the unbaited probe trials con- firm that the feature controlled behavior in these enclosures. In the triangle, all choices on the probe trials were directed to the corner with the black panel (binomial test, $p=.0041)$. In the square, 4 of the 5 rats chose the corner with the panel in the probe test (binomial test, $p=$ .0156).

Individual rats' distributions of choices across the four alternatives in the rectangle are shown in Table 1. On average, they chose the correct corner on $42.67 \%$ of the trials and made a rotational error on $43.33 \%$ of the trials. Searches at N and F were rare, occurring on $7.33 \%$ and $6.67 \%$ of the trials, respectively. The fact that rats were unable to disambiguate the two geometrically correct corners, searching $\mathrm{C}$ and $\mathrm{R}$ equally often, indicates that the disorientation procedure worked and that the rats were not using the smell of the food to find the baited bowl. In the rectangle, all rats chose a geometrically correct corner in the first probe, and 4 rats did so in the second one. GLIM (see Method) determined that across the two tests, choices were distributed nonrandomly $\left[\chi^{2}(1)=\right.$ 7.36], and that there was no effect of successive tests $\left[\chi^{2}(1)=1.49\right]$.

During the consistent test, with the feature in a geometrically correct corner, all 5 rats searched the corner that contained the feature first (binomial test, $p<.001$ ). Thus, prior training to use geometric cues did not prevent use of the feature. During the conflict test, in which the feature was placed in a geometrically incorrect location, again all 5 rats searched in the corner containing the feature first (binomial test, $p<.001$ ). Of course, the rats might have approached the black corner in both tests simply because it was novel in the context of the rectangle. Having approached the black corner, they then might have dug in the nearest bowl. Although the present experiment included no control for this possibility, Experiment 3 in this article does include such a control. Data reported there (Figure 4) show that rats trained to find food in a particular shaped corner of the rectangle in the absence of features and then presented with the black corner panel are more likely to choose the corner with the panel if they have already been trained with it in the square than if it is novel.

In the present experiment, choice of the black panel had been rewarded $100 \%$ of the time in the square and in the triangle, whereas the first geometrically correct choice in

Table 1

Each Rat's Total Choices of Each Kind During Criterion Days and Regular Trials During Probe Days in Experiment 1

\begin{tabular}{ccccc}
\hline \multicolumn{1}{c}{ Rat } & Correct & Rotational & Near & Far \\
\hline 194 & 16 & 11 & 2 & 1 \\
197 & 12 & 14 & 2 & 2 \\
196 & 11 & 12 & 6 & 1 \\
229 & 8 & 17 & 1 & 4 \\
242 & 17 & 11 & 0 & 2 \\
All rats & 64 & 65 & 11 & 10 \\
\hline
\end{tabular}

Note-For definition of kinds of choices, see Figure 1. 
the rectangle was rewarded only $50 \%$ of the time. Thus, the rats' preference for the black panel over a geometrically correct corner could be explained by a difference in associative strength. That is to say, regardless of how the feature and geometry interact during learning, during performance they may compete for control of behavior. An alternative implied by Cheng's (1986) discussion is that they may be used in a hierarchical manner, with the disoriented rats first using the shape of the box to get oriented, and then locating a relevant feature within this geometric framework. The data from this experiment, although limited, do not seem consistent with such an account.

\section{EXPERIMENT 2}

In the test phase of Experiment 1, rats followed the feature regardless of where it was in the rectangle. This behavior could be seen as analogous to that of children who have developed spatial language using it to disambiguate geometry by labeling the correct location in a search task (e.g., Hermer-Vazquez et al., 2001). The rats had learned that the black panel labeled the corner with food in the square and the triangle and then followed it in the rectangle. This account assumes that training outside the rectangle was important in allowing the feature to gain control. Comparison of our results and those of Cheng's (1986) Experiment 3 supports this assumption. In that study, rats trained to choose a geometrically consistent corner of a rectangle marked by a specific panel did not always choose the panel when it conflicted with geometry, although they did choose it sometimes. In our experiment, however, the single black panel within the otherwise white enclosure might simply be so salient that it would control searching more strongly than would geometric cues without special training outside the rectangle. To evaluate this possibility, in Experiment 2 we trained rats to find food in a geometrically distinctive corner of the rectangle, but this corner was marked with the black panel from the outset. The panel was thus a better predictor of the corner with the rewarded bowl than was the left-right position of long and short walls. After the rats reached criterion, control by the panel was tested by moving it to a geometrically incorrect corner, and control by geometry was tested in probes without the panel.

\section{Method}

Subjects. The subjects were 4 experimentally naive male LongEvans rats, 85-94 days of age and weighing 348-366 g at the start of the experiment. They were maintained in the same way as were the rats in Experiment 1.

Apparatus. The apparatus was the rectangular enclosure with the $90^{\circ}$ black corner panel used in Experiment 1 .

Procedure. The rats were shaped to dig for buried Froot Loops as in Experiment 1. They then received training identical to that described for the rectangular enclosure in Experiment 1, except that the correct corner also contained the black panel. Once criterion was reached $(75 \%$ accuracy on the first choice on 4 consecutive days), the rats received 2 days of probe testing, in which one of the final four trials was unrewarded. Provided that performance did not fall below criterion on these days, the probe testing was followed by two test sessions conducted in the same way as were those in Experiment 1 . The 1 rat that fell below criterion on two initial probe testing days was retrained until it met criterion again and then proceeded to unrewarded probes. Each rat had one conflict test like that in Experiment 1 and one no-feature test, in counterbalanced order. In the no-feature test, the rats were placed for $1 \mathrm{~min}$ in the rectangular enclosure without the feature and without reward in any of the bowls. Test sessions were separated by 1 regular training day.

\section{Results and Discussion}

The 4 rats completed criterion plus probe testing within $7,15,18$, and 19 sessions. During both unrewarded probe trials, all rats chose the correct location, demonstrating that they were using the feature and not the odor of the food $\left[\chi^{2}(1)=10.96\right.$; for effect of tests, $\left.\chi^{2}(1)<1.0\right]$.

Figure 2 shows the mean proportions of correct choices, rotational, near, and far errors in the first four 4-day blocks. The majority of errors early in training were rotational, indicating that geometric cues were learned even in the presence of the more informative feature. In the first block, more than $80 \%$ of choices were geometrically correct, but considerably more than half of these were to the corner with the black feature (see Figure 2). This suggests that the rats acquired featural and geometric information in parallel rather than learning geometry first and then learning about the more informative feature, which disambiguated geometry. A firm conclusion on this point would require analyzing acquisition of more animals in smaller blocks.

Final performance was excellent. During the 4 days in which each rat reached criterion and the seven regular trials in each of the 2 probe days (i.e., 46 trials per rat), the rats chose the correct corner on $86.0 \%$ of the trials. Searches at R, N, and F occurred on 9.2\%, 1.6\%, and $3.3 \%$ of the trials, respectively. Thus, even at this stage of training, the number of rotational errors was still almost two thirds of the total errors. There were too few errors during these final days for significance testing to be

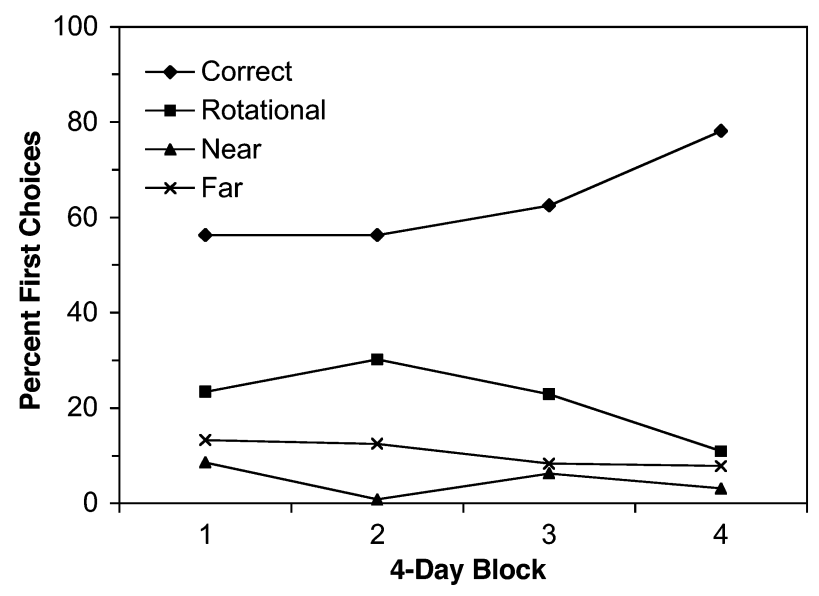

Figure 2. Mean proportions of correct choices and of the three kinds of errors for the first four 4-day blocks of training in Experiment 2. See Figure 1 for specification of the kinds of errors. 
reasonable, so to analyze the distribution of errors, we summed errors across training and probe testing. The 12-50 errors per rat (mean 38.5) were distributed among $\mathrm{R}, \mathrm{N}$, and $\mathrm{F}$ errors in a way that differed significantly from random; Friedman two-way analysis of variance by ranks $\left[\chi_{\mathrm{r}}^{2}(3)=8, p=.0046\right]$. Interestingly, not only did every rat make more rotational than near or far errors, there were also more far than near errors. On average, $56 \%$ of the errors were R, $30 \%$ were $\mathrm{F}$, and $14 \%$ were $\mathrm{N}$.

Although performance during acquisition clearly showed that the rats were learning about the geometry of the enclosure, the sample size was too small to draw strong conclusions from the tests. During the no-feature test 3 out of the 4 rats searched in a geometrically correct location first (binomial test, n.s.). During the conflict test, all 4 of the rats first searched in the corner containing the feature (binomial test, $p=.0039$ ) Thus, although behavior early in training clearly showed that geometry acquired control, by the time of the test the feature controlled choice more strongly than did geometry. This finding is consistent with the results of Experiment 1, in that, for well-trained rats, the black feature was more attractive than geometric cues. Cheng (1986, Experiment 2) and investigators who have tested species other than rats (see introduction to this article) have also found that animals trained in a reference memory task with a distinctive feature in the rewarded corner of a rectangle are influenced to at least some extent by the feature when it conflicts with geometric cues.

\section{EXPERIMENT 3}

Perhaps the most interesting data from Experiment 2 are those from acquisition (Figure 2). Cheng (1986) as well as Golob and Taube (2002) reported that rats learning to find food near a distinctive corner panel in a rectangular enclosure made more rotational errors than other kinds of errors. However, those authors did not report the details of acquisition. As pointed out above, Figure 2 suggests that geometric and featural cues are initially learned in parallel. However, the left-right position of the long versus short side of the rectangle predicts the location of the baited bowl only $50 \%$ of the time, whereas the black corner predicts it perfectly. Thus, the decline of geometric errors as acquisition progresses can be accounted for in associative learning terms by saying that the black panel eventually absorbs more associative strength because it is a better predictor of food. In contrast, the view that environmental shape is processed in an independent cognitive module suggests that although the black panel may gain control with experience, geometry will not lose control. Experiment 2 provided too few data to be conclusive on this point, but its results are consistent with the results of studies showing that geometric cues are not blocked or overshadowed by a conspicuous beacon over the dry platform in a triangular water tank (Pearce et al., 2001) or by landmarks in or near the tank (Hayward et al., 2003). Experiment 3 was designed to discover whether the same lack of blocking would be found in our food-finding task.

Whether animals are looking for food or escaping from water may influence what is learned in tests of the geometric module. Rotating a rat before introducing it to a spatial task (i.e., disorientation, which is key in revealing reliance on geometry) slows rats' landmark learning in food-rewarded but not in water-escape tasks (Dudchenko, Goodridge, Seiterle, \& Taube, 1997; Martin, Harley, Smith, Hoyles, \& Hynes, 1997). This is true even when the two tasks are conducted in the same arena and are similar in difficulty (Gibson, Shettleworth, \& McDonald, 2001). Moreover, one study suggests that disoriented rats are better able to use landmarks to reorient in a rectangular enclosure when they are escaping from water rather than searching for food (Golob \& Taube, 2002). The latter findings should be viewed cautiously, because even in the food-finding task in this study rats did not show very strong control by geometry (see discussion in Golob \& Taube, 2002). Nevertheless, evidence that disorientation interacts with motivation to affect what is learned in spatial tasks means that the findings of Pearce and colleagues (Hayward et al., 2003; Pearce et al., 2001) should be verified in a food-finding task.

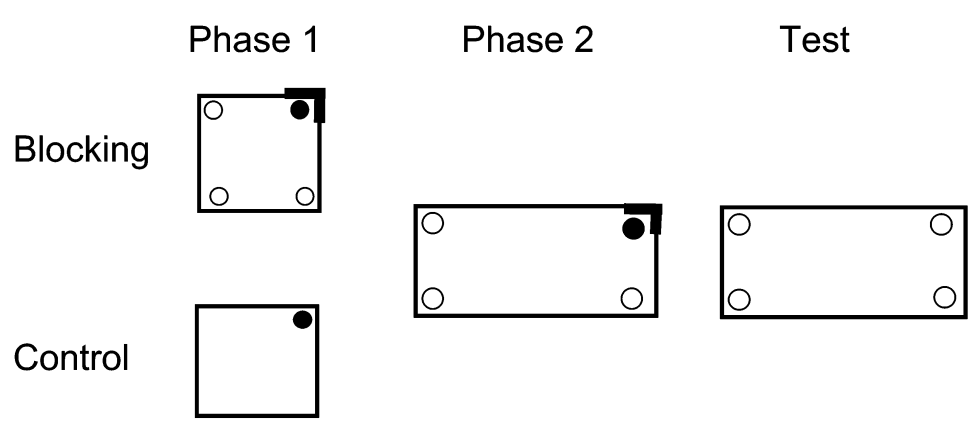

Figure 3. Arrangement of the enclosures and corner panel for the three phases of Experiment 3. The orientation of the enclosure + panel and rewarded bowl within the room was changed from trial to trial as described in the text. Filled circles $=$ bowl containing reward; open circles $=$ unrewarded bowls. 
In Experiment 3, we tested the independence of geometry and visual feature learning using the blocking design illustrated in Figure 3. In Phase 1, the rats in the blocking group were trained in the square enclosure with the black panel marking the corner with food. Concurrently, the rats in the control group had experience digging for food in a single bowl in the unmarked square enclosure. In Phase 2, both groups were trained as the rats in Experiment 2 had been trained, with the feature marking the geometrically consistent rewarded corner in the rectangular enclosure. Finally, all rats were tested in the rectangle without the feature. If local features compete with geometric cues for some analogue of associative strength, the rats in the blocking group should search less often in the geometrically correct corners during the test than should the controls. In addition, during acquisition in the rectangle, the controls, like the rats in Experiment 2 , should make primarily rotational errors, whereas the rats in the blocking group should show a more nearly even distribution of rotational, near, and far errors. In contrast, if geometry and feature learning do not compete, the rats in both groups should make similar proportions of rotational errors in acquisition and choose the geometrically correct corners to the same extent in the test.

\section{Method}

Subjects. The subjects were 12 rats like those in Experiments 1 and 2, maintained in the same way. They were 84-182 days old at the start of the experiment and weighed 330-404 g. Two had previously participated in an unrelated experiment; the others were experimentally naive. They were divided into two groups balanced for age and past experience.

Apparatus. The square and rectangular enclosures from Experiment 1 were used together with the black corner panel and the bowls. The testing room used for Experiment 1 was set up in the same way.

Pretraining. Rats were pretrained to dig in the bowls for Froot Loops as in Experiments 1 and 2.

Phase 1. For the blocking group, this phase consisted of seven 8 -trial sessions of training in the square enclosure with the black corner panel conducted in the same way as was training in the square in Experiment 1. During the first 3 days, the rats were allowed as many choices as was necessary in order to find the Froot Loop; thereafter, the rat was removed from the enclosure after a maximum of two choices. Unrewarded probe trials were given on Trial 6 of Day 4 and on Trial 4 of Day 5.

For the control group, Phase 1 consisted of seven 2-trial sessions in the square enclosure without the black corner panel. A single baited bowl was placed in a corner chosen so that each of the eight possible locations for bowl plus enclosure specified in Experiment 1 was used no more than twice over the 14 training trials. As for the blocking group, if the rat did not find the Froot Loop within 2 min, the Froot Loop was placed on top of the bedding and the rat was given $1 \mathrm{~min}$ to eat it. Trials were conducted in all other respects as for the blocking group, with disorientation before each trial and the enclosure alternately parallel or at $45^{\circ}$ to the walls of the testing room.

Phase 2. The second phase of acquisition for both groups consisted of eight sessions of training in the rectangular enclosure with the black panel present. The food was always in the bowl closest to the panel, which was in a geometrically consistent corner. Sessions were conducted as in Experiment 2.
Test phase. Rats in both groups received three 8-trial test days in the rectangular enclosure without the black panel, with 1 retraining day between test days. Thus, there was a combined total of 13 days in Phase 2 and the test phase. On test days, the first 7 trials were normal training trials, but Trial 8 was conducted with the black panel removed and no reward in any of the bowls. The rat was removed from the apparatus after it had dug in one bowl.

\section{Results and Discussion}

By the end of Phase 1, rats in the blocking group usually chose the corner with the feature first. In the final 2 days of training in the square, $67 \%$ of the first choices were correct. During each of the nonrewarded probes in the square, 4 of 6 rats searched first in the correct corner on their first choice; this overall distribution of choices is significantly different from random $\left[\chi^{2}(3)=9.67\right]$.

Figure 4 shows the proportion of correct first choices and near, far, and rotational errors by both the blocking and control groups during 2-day blocks of training in the rectangle, including the regular trials in the test days. The final test day is omitted because it was not part of a complete 2-day block; performance on regular trials on that day was similar to that in the preceding 2-day block. As in Experiment 1, training with the black panel in the square transferred to the rectangle. During the first 8-trial session in the rectangle, all rats in the blocking group dug first in the correct corner on 6 or more trials, whereas no control rat chose the correct corner first on more than 4 trials. Over the course of training in the rectangle, rats in both the blocking and control groups learned to go directly to the corner with the black panel (Figure 4). The proportions of correct responses in the two groups were compared in a groups $\times$ blocks analysis of variance. The effects of groups and of blocks were significant $[F \mathrm{~s}(1,10)=$ 22.9 and 52.5, respectively]. The block $\times$ group interaction was also significant $[F(1,10)=8.3]$. Planned comparisons showed that, as suggested by Figure 4, the two groups differed significantly in the first block of training but not in later blocks.

Like the animals in Experiment 2, the rats in the control group made more rotational than near or far errors during training in the rectangle with the black corner panel (Figure 4, lower panel). The same was true of the rats in the blocking group (Figure 4, upper panel), contrary to what would be expected if prior training with the feature blocked learning of geometry. Because there were relatively few errors, we analyzed their distribution by summing errors of each type across all regular training trials, including those on days with tests. For the blocking group, a mean of $61 \%$ (range $45 \%-74 \%$ ) of the errors were rotational. For the controls, comparable data were $47 \%(27 \%-61 \%)$. Thus, if anything, the animals in the blocking group tended to make more rather than fewer rotational errors than did controls.

Over the first two tests in the featureless rectangle, $83 \%$ of the first choices made by the rats in the blocking group and $75 \%$ of choices by the controls were to a geometrically correct corner. Analyses of the proportion of rotational and nonrotational choices on these tests were 

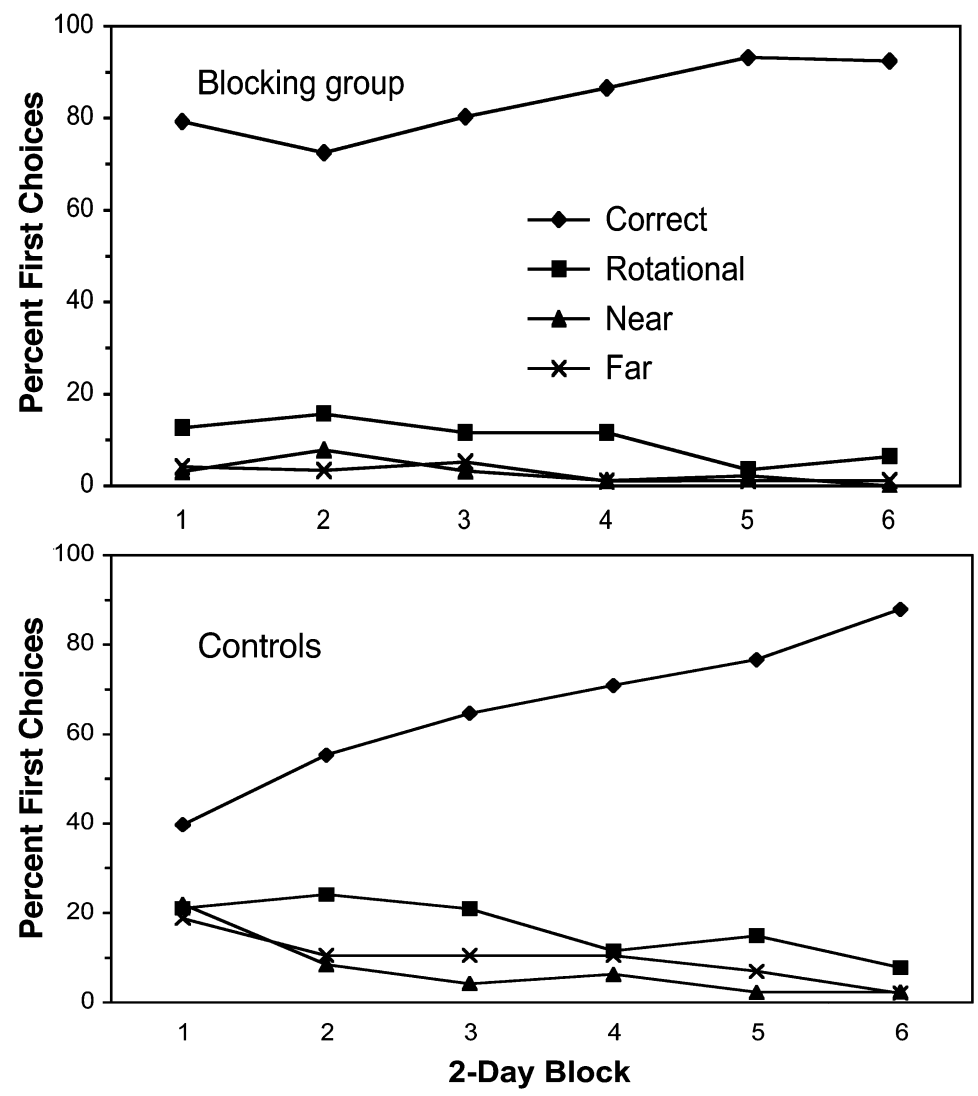

Figure 4. Mean percentage of correct choices and of the three kinds of errors for 2-day blocks of training in the rectangle in Experiment 3. Blocks 5 and 6 include the seven regular trials on the 1 st and 2 nd test days, respectively. See Figure 1 for specification of the kinds of errors.

conducted using GLIM. There was no effect of group $\left[\chi^{2}(1)=.254\right]$. However, there was a significant effect of location (geometrically correct or incorrect) $\left[\chi^{2}(1)=\right.$ 8.71]. On the third test, only half the rats in either group chose a geometrically correct corner first (i.e., both groups' performance fell to chance). The rats may have learned that they were never rewarded in the featureless rectangle. Nevertheless, a GLIM test on all 3 of the test days together still indicated that the overall distribution of choices was significantly different from random and did not differ between the groups $\left[\chi^{2}(1)=4.61\right.$ and 0.23 , respectively].

The results of this experiment provide no evidence that prior training with the black feature blocked learning about the position of food with respect to the shape of the rectangular enclosure. The group differences at the beginning of Phase 2 show that training with the feature in the square enclosure transferred to the rectangle. Nevertheless, the blocking group made predominantly rotational errors during training in the rectangle and chose geometrically correct corners in the tests to the same extent as did the controls. The pattern of errors by the blocking group shows that they were learning about geometric cues in the rectangle from the very beginning of Phase 2, even though they had already learned to use the black panel, which was a more valid cue to the location of food. These data therefore give even stronger support to the view that learning overall geometry is independent of learning local features than do the data of Pearce and his colleagues (2001; Hayward et al., 2003). In the triangular enclosure that Pearce and colleagues used, geometry was just as predictive of the location of the goal as were the beacons and landmarks, but here it was not as good a predictor as the local feature was. No researchers have yet reported an attempt to test for cue competition by training geometry first and then adding a beacon or landmarks. Because geometric cues do not appear to lose control as a more predictive feature is learned, our data are consistent with Cheng's (1986) discussion of the geometric module in suggesting that blocking would not be found with such a design.

\section{GENERAL DISCUSSION}

Whether or not spatial learning is acquired as associative learning is, with different sources of information 
competing for some analogue of associative strength, is a subject of some debate (Mackintosh, 2002; Shettleworth, 1998). The most prominent competing view is that spatial information is acquired as an integrated cognitive map (O'Keefe \& Nadel, 1978). Another possibility consistent with neurobiological evidence is that separable spatial memory systems, such as those supporting response learning, cue or beacon learning, and landmark learning, acquire information simultaneously and in parallel (see White \& McDonald, 2002). Only the view that spatial learning is like associative learning predicts cue competition effects such as overshadowing and blocking. Thus, reports that when rats are trained in water-escape tasks beacons or landmarks fail to overshadow or block learning based on the shape of the tank (Hayward et al., 2003; Pearce et al., 2001) are of considerable interest.

The purpose of the experiments reported in this article was to examine interactions of geometry and visual feature learning in the same task in which the geometric module was originally studied - namely, when rats search for food in a rectangular enclosure. Like Pearce and his colleagues (Hayward et al., 2003; Pearce et al., 2001) in their studies of rats in a triangular water tank, we found no evidence that a distinctive feature near the goal blocks learning the location of the goal relative to the shape of the enclosure. Because we used a rectangular enclosure, in which rats could make rotational errors, and measured a discrete choice response (digging in a bowl in a particular corner), we could document learning based on the geometry of the enclosure throughout acquisition as well as in unrewarded tests at asymptote. Even rats that had learned to use the more informative black corner panel in Experiment 3 made primarily rotational errors from the outset of training with it in the rectangle, and the proportion of rotational errors was not less than that for the controls (see Figure 4). These data constitute striking evidence that cues to the shape of the enclosure do not compete with learning more local cues such as the black corner panel. But this need not mean that geometry and feature learning are independent. Cheng (1986) suggested that geometry is necessarily primary. Only after getting oriented by means of the geometric module do animals acquire nongeometric information, which is coordinated with the reference frame provided by geometry. The results from Cheng's working memory task are consistent with this view, in that rats learned the geometric location of buried food after a single exposure but not its location with respect to features in the box. However, a difference in learning rate is also compatible with parallel but independent acquisition of geometric and featural information, and this interpretation is more consistent with our data. In particular, the data from the blocking group in Experiment 3 show that rats that have already acquired relevant featural information still learn about the location of the goal with respect to local geometry, which seems to be the opposite of the sequence of learning proposed by Cheng.
In studies of control by geometry, beginning with those of Cheng (1986), the featural cues used have sometimes been distinctive panels covering whole walls of a rectangular enclosure and sometimes distinctive panels or objects in the corners. These seem to be treated similarly, by both the subjects and the researchers (see Cheng \& Newcombe, in press). However, in discussions of spatial learning, beacons, or cues at goals, are typically distinguished from landmarks, or cues distal to goals. Beacons are arguably computationally less demanding cues than landmarks, because using a landmark to find a goal requires encoding and remembering both distance and direction information (see Shettleworth, 2000). A beacon at the dry platform was the nongeometric cue in the experiments by Pearce et al. (2001), but landmarks consisting of objects distal to the dry platform were used by Hayward et al. (2003). Both revealed no evidence of blocking or overshadowing in a triangular water tank. In our study, as in related studies with a goal near a distinctively marked corner, the "feature" was displaced from the goal itself, but it nevertheless may have functioned like a beacon because a rat simply had to head toward it to find the baited bowl. Future studies of interactions between geometric and nongeometric information may need to consider a possible role for differences among kinds of nongeometric information.

The view of spatial learning supported by the present study and related work (see Mackintosh, 2002; Shettleworth, 2000) is that spatial behavior is supported by a number of distinct information processing and acquisition systems rather than by an integrated cognitive map. For vertebrates performing short-distance navigation as in laboratory settings, these subsystems include dead reckoning, beacon (or cue) learning, landmark learning, response or habit learning, and learning the overall shape of the local space formed by enclosing surfaces. To the extent that each of these has distinct computational demands and perhaps neurological substrates, spatial information processing is modular. Whether any candidate module fulfills all the criteria for cognitive modularity originally set out by Fodor (1983), or whether these criteria even continue to be useful, is debatable (see Cheng \& Newcombe, in press; Coltheart, 1999). In any case, rather than ask whether a possible geometric module is impenetrable to other kinds of spatial information, it may be more productive to ask how distinguishable kinds of spatial information interact during acquisition and performance and what determines which information takes priority. Investigations to date seem to show that whereas some sources of spatial information, such as beacons and landmarks, compete for predictive value, others are learned in parallel or may interact in other ways. Geometry may take precedence in disoriented animals, but it is not clear that this makes it any more modular than other computationally distinct kinds of spatial information. A task for the future is to better characterize the various spatial subsystems and the ways in which they interact during learning and performance. 


\section{REFERENCES}

Benhamou, S., \& Poucet, B. (1998). Landmark use by navigating rats (Rattus norvegicus): Contrasting geometric and featural information. Journal of Comparative Psychology, 112, 317-322.

Chamizo, V. D. (2003). Acquisition of knowledge about spatial location: Assessing the generality of the mechanism of learning. Quarterly Journal of Experimental Psychology, 56B, 102-113.

CHENG, K. (1986). A purely geometric module in the rat's spatial representation. Cognition, 23, 149-178.

Cheng, K., \& NewCOMBE, N. S. (in press). Is there a geometric module for spatial orientation? Squaring theory and evidence. Psychonomic Bulletin \& Review.

Coltheart, M. (1999). Modularity and cognition. Trends in Cognitive Sciences, 3, 115-120.

DudChenko, P. A., Goodridge, J. P., Seiterle, D. A., \& Taube, J. S. (1997). Effects of repeated disorientation on the acquisition of spatial tasks in rats: Dissociation between the appetitive radial arm maze and aversive water maze. Journal of Experimental Psychology: Animal Behavior Processes, 23, 194-210.

FoDOR, J. A. (1983). The modularity of mind. Cambridge, MA: MIT Press.

Francis, B., Green, M., \& Payne, C. (Eds.) (1993). The GLIM system: Statistical software for generalized linear interactive modelling. Oxford: Oxford University Press, Clarendon Press.

Gibson, B. M., Shettleworth, S. J., \& McDonald, R. J. (2001). Finding a goal on dry land and in the water: Differential effects of disorientation on spatial learning. Behavioural Brain Research, $\mathbf{1 2 3}$, 103-111.

Golob, E. J., Stackman, R. W., Wong, A. C., \& Taube, J. S. (2001). On the behavioral significance of head direction cells: Neural and behavioral dynamics during spatial memory tasks. Behavioral Neuroscience, 115, 285-304.

GoLOB, E. J., \& TAUBE, J. S. (2002). Differences between appetitive and aversive reinforcement on reorientation in a spatial working memory task. Behavioural Brain Research, 136, 309-316.

GouteuX, S., Thinus-Blanc, C., \& VAUClaIR, J. (2001). Rhesus monkeys use geometric and nongeometric information during a reorientation task. Journal of Experimental Psychology: General, 130, 505519.

Hayward, A., McGregor, A., Good, M. A., \& Pearce, J. M. (2003). Absence of overshadowing and blocking between landmarks and the geometric cues provided by the shape of a test arena. Quarterly Journal of Experimental Psychology, 56B, 114-126.

HeRMER, L., \& SPELKE, E. (1996). Modularity and development: The case of spatial reorientation. Cognition, 61, 195-232.

Hermer-Vazquez, L., Moffet, A., \& MUNKholm, P. (2001). Language, space, and the development of cognitive flexibility in humans: The case of two spatial memory tasks. Cognition, 79, 263-299.

Hermer-Vazquez, L., Spelke, E. S., \& Katsnelson, A. S. (1999). Sources of flexibility in human cognition: Dual-task studies of space and language. Cognitive Psychology, 39, 3-36.

Kamin, L. J. (1969). Predictability, surprise, attention, and conditioning. In B. A. Campbell \& R. M. Church (Eds.), Punishment and aversive behavior (pp. 279-296). New York: Appleton-Century-Crofts.

Kelly, D. M., \& SPETCH, M. L. (2001). Pigeons encode relative geom- etry. Journal of Experimental Psychology: Animal Behavior Processes, 27, 417-422.

Kelly, D. M., Spetch, M. L., \& Heth, C. D. (1998). Pigeons' (Columba livia) encoding of geometric and featural properties of a spatial environment. Journal of Comparative Psychology, 112, 259-269.

Learmonth, A. E., Nadel, L., \& Newcombe, N. S. (2002). Children's use of landmarks: Implications for modularity theory. Psychological Science, 13, 337-341.

LeARmonth, A. E., Newcombe, N. S., \& Huttenlocher, J. (2001). Toddlers' use of metric information and landmarks to reorient. Journal of Experimental Child Psychology, 80, 225-244.

MACKINTOSH, N. J. (2002). Do not ask whether they have a cognitive map, but how they find their way about. Psicológica, 23, 165-185.

Margules, J., \& Gallistel, C. R. (1988). Heading in the rat: Determination by environmental shape. Animal Learning \& Behavior, 16, 404-410.

Martin, G. M., Harley, C. W., Smith, A. R., Hoyles, E. S., \& Hynes, C. A. (1997). Spatial disorientation blocks reliable goal location on a plus maze but does not prevent goal location in the Morris maze. Journal of Experimental Psychology: Animal Behavior Processes, 23, 183-193.

O'KeEFE, J., \& NADEL, L. (1978). The hippocampus as a cognitive map. Oxford: Oxford University Press, Clarendon Press.

Pavlov, I. P. (1927). Conditioned reflexes (G. V. Anrep, Trans.). London: Oxford University Press.

Pearce, J. M., Ward-Robinson, J., Good, M., Fussell, C., \& Aydin, A. (2001). Influence of a beacon on spatial learning based on the shape of the test environment. Journal of Experimental Psychology: Animal Behavior Processes, 27, 329-344.

RESCORLA, R. A., \& WAGNER, A. R. (1972). A theory of Pavlovian conditioning: Variations in the effectiveness of reinforcement and nonreinforcement. In A. H. Black \& W. F. Prokasy (Eds.), Classical conditioning II: Current theory and research (pp. 64-99). New York: Appleton-Century-Crofts.

ShetTleworth, S. J. (1998). Cognition, evolution, and behavior. New York: Oxford University Press.

ShetTLEWORTH, S. J. (2000). Modularity and the evolution of cognition. In C. Heyes \& L. Huber (Eds.), The evolution of cognition (pp. 43-60). Cambridge, MA: MIT Press.

Sovrano, V. A., Bisazza, A., \& Vallortigara, G. (2003). Modularity as a fish (Xenotoca eiseni) views it: Conjoining geometric and nongeometric information for spatial reorientation. Journal of Experimental Psychology: Animal Behavior Processes, 29, 199-210.

Vallortigara, G., Zanforlin, M., \& Pasti, G. (1990). Geometric modules in animals' spatial representations: A test with chicks (Gallus gallus domesticus). Journal of Comparative Psychology, 104, 248-254.

Wang, R. F., Hermer, L., \& SpelKe, E. (1999). Mechanisms of reorientation and object localization by children: A comparison with rats. Behavioral Neuroscience, 113, 475-485.

White, N. M., \& MCDonaLD, R. J. (2002). Multiple parallel memory systems in the brain of the rat. Neurobiology of Learning \& Memory, 77,, $125-184$.

(Manuscript received November 10, 2003; revision accepted for publication February 17, 2004.) 